\section{Giant retinal tear management: an Asian experience}

SY Lee, SG Ong, DWK Wong and CL Ang

degeneration. The remaining types occur secondary to trauma (20\%) or along the posterior edge of chorioretinal degeneration or scarring. ${ }^{2,3}$ The management of GRTs has been challenging to vitreoretinal surgeons.

Improvements in instrumentation, introduction of perfluorocarbon liquids, and modern surgical techniques have improved the rate of surgical success.

We report our 15-year experience of managing retinal detachments secondary to GRTs at the Singapore National Eye Centre (SNEC). Myopia was present in 81 eyes $(63.3 \%)$ with mean myopia -7.56 dioptres sphere, DS (-1.00 to $-27.00 \mathrm{DS})$. All eyes underwent pars plana vitrectomy. Scleral buckling was done in 90 eyes $(70.3 \%)$ and lens removal in 49 of 95 phakic eyes $(51.2 \%)$. Total 84 eyes $(84.8 \%)$ were re-attached at 6 months after vitrectomy. This was achieved with one surgery in 71 eyes (71.7\%). The mean number of surgeries for reattachment was 1.19 surgeries. At 6 months, 41 eyes $(41.4 \%)$ regained $20 / 40$ or better vision. A total of 47 vitreoretinal pathologies were seen in the fellow eyes of the 124 patients, including 4 with GRTs.

Conclusion Surgical success for GRT can be achieved with good visual outcome in $84.8 \%$ after a mean of 1.19 surgeries. The fellow eyes are however at risk of pathologies and should be followed-up in the long term.

Eye (2009) 23, 601-605; doi:10.1038/eye.2008.48; published online 29 February 2008

Keywords: retinal detachment; giant retinal tear; myopia; Chinese; vitrectomy; lattice

\section{Introduction}

A giant retinal tear (GRT) is a full-thickness retinal break of at least $90^{\circ}$ in extent circumferentially. ${ }^{1}$ Schepens ${ }^{2,3}$ described the majority (70\%) of GRTs to be idiopathic, as these occur in the absence of chorioretinal

\section{Methods}

All vitreoretinal surgeries performed at the SNEC are coded by diagnosis and audited. All cases that were coded as GRTs were identified from the database and the case records of patients with at least 6 months follow-up post-surgery were pulled for retrospective review. Analysis of the patient demographics, ie age, race, and sex, was done. The ophthalmic data studied included severity of myopia, previous ocular surgery, previous ocular trauma, lens status, visual acuity at presentation, extent of GRT, surgery performed, and post-operative outcomes in terms of anatomical and functional results and complications. The status of the fellow eyes was also noted.

\section{Results}

A total of 128 eyes of 124 patients were managed at SNEC for the diagnosis of retinal detachment secondary to GRT between January 1991 and December 2005. The post-operative follow-up ranged from 1 month to 15 years. Of them, 99 eyes of 96 patients achieved follow-up of at least 6 months after surgery (mean 63.38 months, range 6-180 months) and we report on the functional and anatomical outcomes of the 99 eyes.
Singapore National Eye Centre, Singapore, Singapore

Correspondence: $\mathrm{Dr}$ CL Ang, Singapore National Eye Centre,

11 Third Hospital Avenue, Singapore 168751, Singapore

Tel: + 6563228335 ;

Fax: + 6562263395

E-mail: ang.chong.lye@ snec.com.sg

Received: 12 October 2007 Accepted in revised form: 29 January 2008 Published online: 29 February 2008 
Of the 124 patients, the mean age was 39.6 years with a range of 8-72 years. There was a male preponderance $(91 \%)$ with only 11 women. The majority of the patients were Chinese (80.6\%; Figure 1). A history of myopia was recorded for 81 eyes $(63.3 \%)$ of 78 patients $(62.9 \%)$, of which 52 eyes had myopia of -6.00 dioptres sphere (DS) or greater. The mean myopia was -7.56 DS (range -1.00 to $-27.00 \mathrm{DS}$ ). There were 78 right eyes and 50 left eyes. Seventeen eyes had suffered some ocular trauma prior to presentation.

Total 95 eyes were phakic at presentation. Cataract surgery had been done in 33 eyes previously (Figure 2). Eight of these eyes had either suffered intra-operative posterior capsular rupture with vitreous loss or had undergone a Nd:YAG laser capsulotomy for posterior capsular opacification.

The majority of eyes had GRTs of $90-180^{\circ}$ in extent (72 eyes, 56.3\%; Figure 3). Twelve eyes $(9.4 \%$ ) had primary proliferative vitreoretinopathy at presentation.

All 128 eyes underwent three-port pars plana vitrectomy with vitreous base trimming, perfluorocarbon liquids to unfold the rolled-over flap of the GRT and air-fluid exchange. A scleral buckle was used in 90 eyes (70.3\%). Lens removal was performed in 49 of 95 phakic

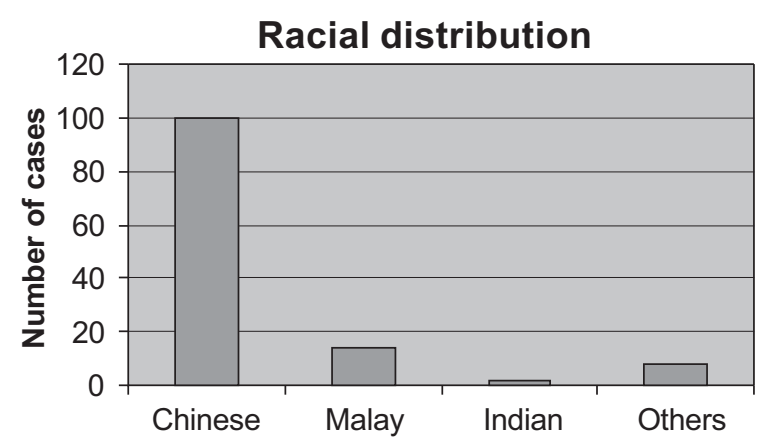

Figure 1 Racial distribution.

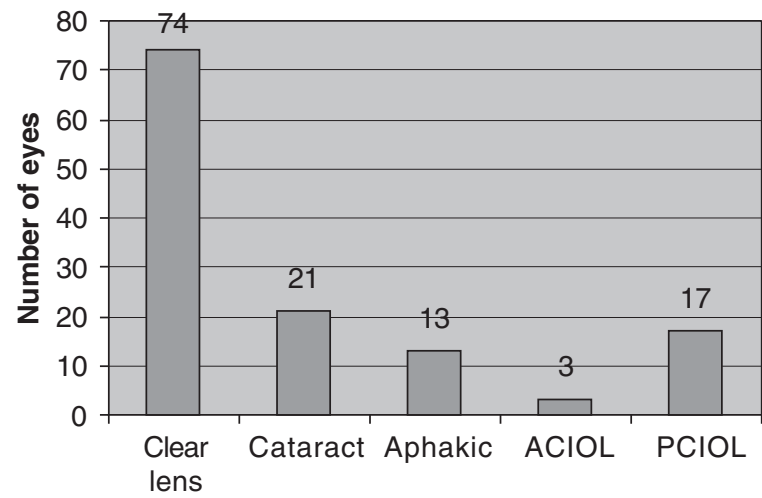

Figure 2 Lens status at presentation. eyes $(51.2 \%)$. Of these, lensectomy was done in 41 eyes (83.7\%), phacoemulsification in seven eyes (14.3\%) and extracapsular cataract extraction in one eye $(2 \%)$.

A primary intraocular lens was implanted in two eyes. The most commonly used tamponade agent was perfluoropropane (C3F8), followed by silicone oil (Figure 4).

The functional and anatomical outcomes were analysed for the 99 eyes that achieved at least 6 months follow-up after surgery, mean 63.38 months (range 6-180 months). Total 84 eyes (84.8\%) were successfully re-attached at 6 months. This was achieved after one surgery in 71 eyes (71.7\%). In total, 13 eyes (13.1\%) required repeat surgeries. Surgical success was achieved after 1.19 surgeries (for all 99 eyes). There was surgical failure in 15 eyes (15.2\%).

At 6 months, 41 eyes (41.4\%) regained $20 / 40$ or better vision and 70 eyes $(70.7 \%$ ) achieved $20 / 200$ or better acuity. The pre- and post-operative visual acuities were converted to $\log M A R$ and charted in a scatter plot in Figure 5.

Several complications were experienced in this series. In the immediate post-operative period, one eye suffered a gross hyphaema that obscured any view of the retina

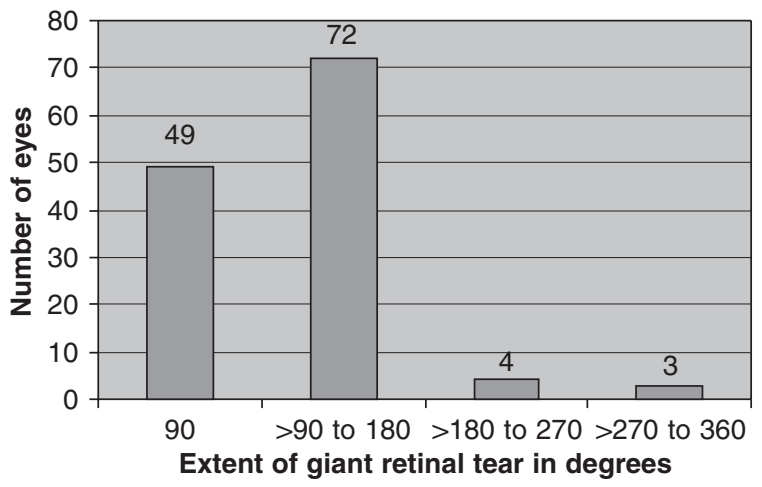

Figure 3 Extent of giant retinal tears (GRT).

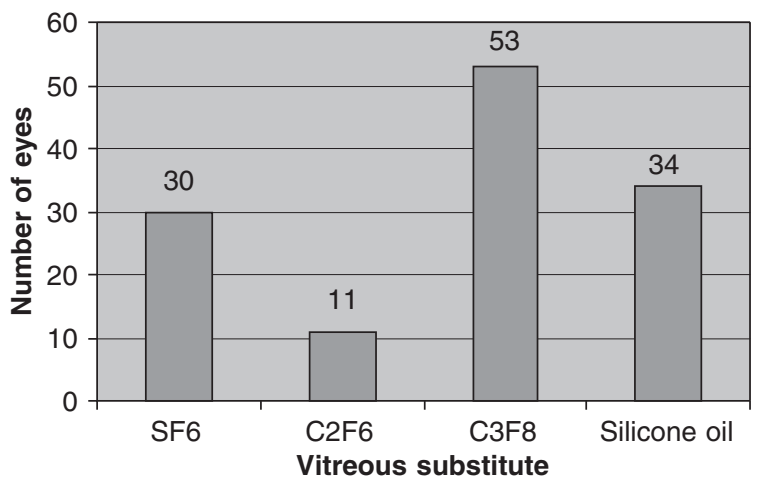

Figure 4 Vitreous substitute used. 


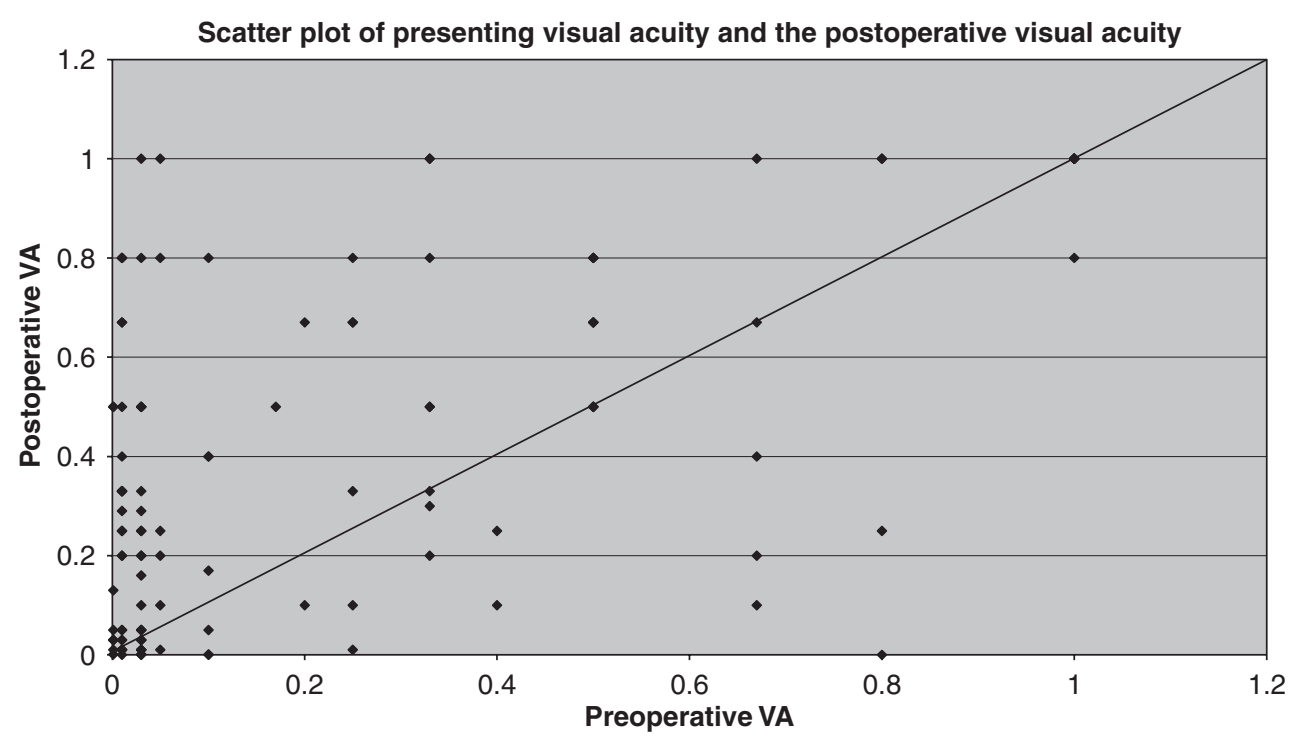

Figure 5 Visual outcome after surgery, pre- vs post-operative visual acuities (Snellen acuities were converted to logMAR).

and one eye required removal of silicone oil due to overfill. Slippage was noted in three eyes but these were limited and none required additional intervention. Proliferative vitreoretinopathy occurred in 10 eyes postoperatively. Cataracts developed in 17 eyes, all of which underwent surgery by phacoemulsification. Silicone oil emulsification occurred in one eye. Three eyes required anti-glaucoma therapy and another developed neovascular glaucoma. Band keratopathy occurred in one eye, epithelial downgrowth in another and one eye required a penetrating keratoplasty for corneal decompensation. Three eyes eventually became phthisical.

Of the 124 patients, there were three fellow eyes that were prosthetic after failed surgery for retinal detachments several years prior. Seventeen other fellow eyes had retinal detachments, of which four were due to GRTs and were included in this review, ie four patients suffered bilateral GRTs (3.2\%). In total, 20 patients $(16.1 \%)$ had retinal detachments in the fellow eyes. Lattice degeneration was observed in 15 fellow eyes $(12.1 \%)$ and 12 fellow eyes $(9.7 \%)$ were treated for retinal tears by cryopexy or laser photocoagulation. Sympathetic ophthalmia occurred in one fellow eye.

\section{Discussion}

Giant retinal tears are defined as retinal tears that extend $90^{\circ}$ or more around the circumference of the globe. The majority of these cases are idiopathic in nature $(60-80 \%)$ with trauma accounting for approximately $20 \% .{ }^{1-3}$ Other predisposing causes include chorioretinal degenerations in which the GRTs occur along the posterior edges as

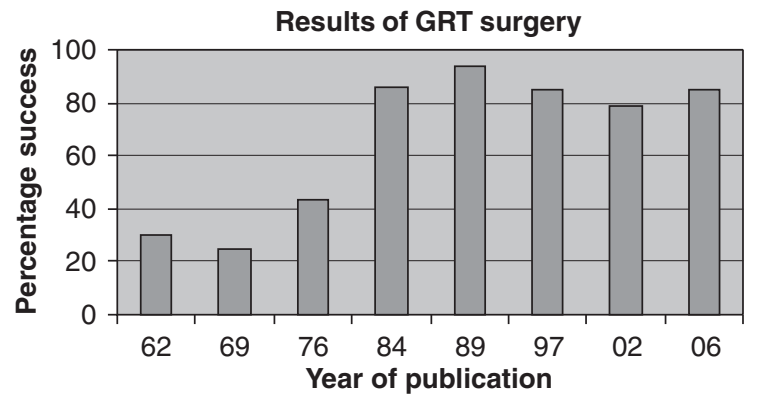

Figure 6 Results of giant retinal tears (GRT) surgery.

well as secondary to surgical trauma such as anterior vitrectomy for posterior capsular rupture and 'dropped' nuclei during cataract surgery, ${ }^{4}$ and underlying syndromes such as Marfan, Ehlers-Danlos and Wagner-Stickler syndromes. There is a predominance of men $(76-85 \%),{ }^{1,5}$ and an association with high myopia in up to $40 \%{ }^{1}$ Up to $13 \%$ of bilateral involvement has been reported. ${ }^{5}$

The GRTs encountered in our review are associated with high myopia (40.6\%) and tend to occur in relatively young Chinese men (mean age 39.6 years). This racial distribution is in keeping with the local population as Chinese form three quarters of the population in Singapore. Furthermore, the Chinese have the highest prevalence of myopia locally. ${ }^{6}$

The results of surgery for retinal detachments and GRTs have vastly improved over the years (Figure 6), ${ }^{2,7-12}$ particularly after the introduction of perfluorocarbon liquids by Chang, enabling controlled, atraumatic unfolding of the retinal flap of the GRT. ${ }^{11}$ Most centres 
now report success rates of at least $70 \%$. In our centre, we were able to achieve surgical success in $84.8 \%$ after a mean of 1.19 surgeries, of which $71.7 \%$ were re-attached after only one operation. Visual acuities of 20/40 or better were achieved in $41.4 \%$.

In cases in which the GRT is limited in extent, ie $90-120^{\circ}$, lens-sparing surgery is an option. However, the lens should be removed if it is cataractous, if the GRT is $180^{\circ}$ or greater, or if proliferative vitreoretinopathy is present. In our series, $51.2 \%$ patients underwent lens surgery, majority by lensectomy. Successful surgical management of GRTs requires good vitreous base shaving, with attention paid to the vitreous attachments at the ends of the giant break. In the aphakic state, this aim can be achieved as there is better visualization of the peripheral retina and the ora.

The use of an encirclage combined with vitrectomy for managing GRTs has been a point of contention. Some surgeons believe that the indent from the buckle distorts the shape of the eyeball and can increase the risk of slippage, particularly intraoperatively. ${ }^{13}$ The encirclage, on the other hand, provides support to the vitreous base and the attached retina, especially at both ends of the GRT, and hence can reduce the risk of re-detachments. ${ }^{11}$ A buckle should also be used in the presence of proliferative vitreoretinopathy. In our series, 90 eyes (70.3\%) received scleral buckling with encirclage.

Cryotherapy increases the risk for PVR and so photocoagulation is preferred. ${ }^{14}$ At SNEC, the preferred practice is to perform photocoagulation along the edge of the GRT combined with cryotherapy applied to the horns of the GRT. We generally do not extend the photocoagulation $360^{\circ}$ around the normal retina, which has been recommended by several authors, to increase retinal adherence and reduce failures. ${ }^{15}$

Over the past 15 years, the surgical technique at SNEC had evolved, with a tendency to encircle the eye less frequently, a greater usage of long-acting gases instead of silicone oil, and lens-sparing surgery, unless the GRT was too extensive.

Retinal detachments secondary to GRTs tend to be at higher risks of developing PVR, with high re-detachment rates and, hence, high re-operation rates. ${ }^{16} \mathrm{We}$ experienced a rate of re-operations of $13.1 \%$ and failure rate of $15.2 \%$, not dissimilar to reports by other surgeons.

The majority of the complications encountered in this series are largely known complications of vitrectomies. The unusual complication here, being the single case of sympathetic ophthalmia in a fellow eye.

It is likely in eyes with abnormal vitreoretinal adhesions to be predisposed to retinal tears and detachments. This tends to be present bilaterally and the fellow eyes are at risk of significant vitreoretinal pathology (12 and 50\%). ${ }^{5}$ In this series, a total of 47 patients $(37.1 \%)$ had fellow eye retinal pathology. These included 20 fellow eyes $(16.1 \%)$ that developed or had suffered prior retinal detachments, of which 4 were due to GRTs (3.2\%). Lattice degeneration was present in 15 fellow eyes $(12.1 \%)$ and retinal tears in 12 eyes $(9.7 \%)$. Prophylactic treatment of the fellow eye is controversial. Some authors recommend that the asymptomatic fellow eye should be treated prophylactically with laser photocoagulation, cryotherapy, or scleral buckling. ${ }^{17,18}$ However, there has been no evidence to suggest that any one of these is superior to the other. At SNEC, we routinely perform photocoagulation to areas of lattice and retinal breaks in the fellow eyes of GRTs. We do not recommend laser to normal areas of the retina.

The prognosis of managing GRTs has improved over the recent years with better instrumentation and surgical visualization. Surgical success can be achieved with good visual outcome in $84.8 \%$ after a mean of 1.19 surgeries, with good functional visual outcome. In our series, 70 eyes $(70.7 \%)$ achieved $6 / 60$ or better acuity at 6 months, of which 41 eyes had final acuities of 20/40 or better. The fellow eyes of these cases are however at risk of retinal tears and detachments and should be followed-up in long term; and while prophylactic treatment is controversial it may be a good option to consider in the presence of lattice degeneration and retinal breaks.

\section{References}

1 Schepens CL. Retinal Detachment and Allied Diseases. WB Saunders: Philadelphia, 1983, pp 520-528.

2 Schepens CL, Freeman HM. Current management of giant retinal breaks. Trans Am Acad Ophthalmol Otolaryngol 1967; 71: 474-487.

3 Schepens CL, Dobble JG, McMeel JW. Retinal detachments with giant breaks: preliminary report. Trans Am Acad Ophthalmol, Otolaryngol 1962; 66: 471-479.

4 Aaberg TM, Rubsamen PE, Flynn Jr HW, Chang S, Mieler WF, Smiddy WE. Giant retinal tears as a complication of attempted removal of intravitreal lens fragments during cataract surgery. Am J Ophthalmol 1997; 124: 222-226.

5 Freeman HM. Current management of giant retinal breaks and fellow eyes. In: Ryan SJ (ed). Retina, 2nd edn, vol. 3 Mosby: St Louis, 1994, pp 2313-2328.

6 Wong TY, Foster PJ, Hee J, Ng TP, Tielsch JM, Chew SJ et al. The prevalence and risk factors for refractive errors in adult Chinese residents in Singapore. Invest Ophthalmol Vis Sci 2000; 41: 2486-2494.

7 Norton EWD, Aaberg T, Fung W, Curtin VT. Giant retinal tears. Clinical management with intravitreal air. Am J Ophthalmol 1969; 68: 1011-1021.

8 Machemer R, Allen AW. Retinal tears 180 degrees and greater. Management with vitrectomy and intravitreal gas. Arch Ophthalmol 1976; 94: 1340-1346.

9 Lever PK, Lean JS. Management of giant retinal tears using vitrectomy and silicone oil/fluid exchange. A preliminary report. Trans Ophthalmol Soc UK 1981; 101: 189-191. 
10 Chang S, Lincoff H, Zimmerman NJ, Fuchs W. Giant retinal tears. Surgical techniques and results using perfluorocarbon liquids. Arch Ophthalmol 1989; 107: 761-766.

11 Kertes PJ, Wafapoor H, Peyman GA, Calixto Jr N, Thompson $\mathrm{H}$. The management of giant retinal tears using perfluoroperhydrophenanthrene: a multicentre case series. Vitreon Collaborative Study Group. Ophthalmology 1997; 104: 1159-1165.

12 Scott IU, Murray TG, Flynn Jr HM, Feuer WJ, Schiffman JC, Perfluoron Study Group. Outcomes and complications associated with giant retinal tear management using perfluoro-n-octane. Ophthalmology 2002; 109: 1828-1833.

13 Kreiger AE, Lewis $\mathrm{H}$. Management of giant retinal tears without scleral buckling. Use of radical dissection of the vitreous base and perfluoro-octane and intraocular tamponade. Ophthalmology 1992; 99: 491-497.
14 Ghosh YK, Banerjee S, Savant V, Kotamarthi V, Benson MT, Scott RA et al. Surgical treatment and outcome of patients with giant retinal tears. Eye 2004; 18(10): 996-1000.

15 Ambresin A, Wolfensberger TJ, Bovey EH. Management of giant retinal tears with vitrectomy, internal tamponade and peripheral 360 degrees retinal photocoagulation Retina 2003; 23(5): 622-628

16 Glaser BM. Treatment of giant retinal tears combined with proliferative vitreoretinopathy. Ophthalmology 1986; 93(9): 1193-1197.

17 Freeman HM. Fellow eye of giant retinal breaks. Trans Am Ophthalmol Soc 1978; 76: 343-382.

18 Wolfensberger TJ, Aylward GW, Leaver PK. Prophylactic 360 degrees cryotherapy in fellow eyes of patients with spontaneous giant retinal tears. Ophthalmology 2003; 110(6): 1175-1177. 